\title{
LES ACTIVITES EXPERIMENTALES \\ DANS LE MANUEL SCOLAIRE ET LES PRATIQUES DE CLASSE DES SCIENCES PHYSIQUES DE TROISIEME ANNEE DU SECONDAIRE COLLEGIAL AU MAROC : ETUDE DE CAS ${ }^{i}$
}

\author{
Mohammed Abid ${ }^{1,2 i i}$, \\ Amina Boulahoual', \\ Abdeslem Daif ${ }^{2}$, \\ Ali Ouasri ${ }^{1}$ \\ ${ }^{1}$ Laboratoire de la Recherche Scientifique \\ et Innovation Pédagogique (ReSIP), \\ Centre Régional des Métiers de l'Education \\ et de la Formation, \\ Madinat Al Irfane, Souissi, BP 6210, \\ Rabat, Maroc \\ ${ }^{2}$ Laboratoire des Matériaux Avancés et \\ Génie des Procédés, \\ Faculté des Sciences Univesité Ibn Tofail, BP133, \\ Kénitra, Maroc
}

\section{Résumé :}

Cet article porte sur les activités expérimentales et documentaires dans le manuel scolaire des sciences physiques de troisième année du secondaire collégial, les pratiques expérimentales des enseignants et l'effet de ces activités sur l'apprentissage de concepts physiques et chimiques par les élèves. La représentation générale du manuel scolaire est développée à l'aide d'une grille appropriée. L'analyse montre la place importante des activités expérimentales proposées dans ce manuel, conformément aux orientations pédagogiques officielles marocaines. La partie empirique est effectuée à l'aide d'un questionnaire adressé aux enseignants et un test dédié aux élèves en vue d'étudier les pratiques expérimentales des enseignants et ses effets sur l'apprentissage des élèves de certains concepts physiques. Selon les enseignants, la quasi-totalité (90\%) des activités expérimentales conçues dans le manuel scolaire ne sont pas réalisées, à cause du manque de matériel expérimental et de sureffectif des classes ; ce qui affecte le taux de réalisation des expériences programmées et les pratiques expérimentales des enseignants. Les activités expérimentales rarement effectués se déroulent sous forme des expériences de cours, réalisées par l'enseignant lui-même. Les Technologies d'Information et de Communication (TIC) ne sont pas très utilisées par les enseignants, qui par ailleurs,

\footnotetext{
' EXPERIMENTAL ACTIVITIES IN THE TEXTBOOK AND CLASSROOM PHYSICAL SCIENCE PRACTICES OF THIRD YEAR MIDDLE SCHOOL IN MOROCCO: CASE STUDY

ii Correspondence: email profabidmed@gmail.com
} 
pensent qu'elles seraient un bon moyen pour complémenter les expériences. L'analyse des réponses des élèves montre qu'ils ne maitrisent pas les concepts fondamentaux en sciences physiques lorsqu'ils n'ont pas l'occasion d'observer dans la pratique ou dans leur vie des objets rattachés à ces concepts.

Mots clés : activité expérimentale, manuel scolaire, enseignement, sciences physiques

\begin{abstract}
:
This work is interested in the experimental activities in the physical sciences textbook of the third year of middle school cycle, the experimental practices of teachers, as well as the impact of these activities on the learning of certain fundamental concepts in physical sciences by the pupils. The general representation of the textbook is developed using an appropriate grid. The analysis showed the important place of proposed experimental activities in this manual, in accordance with the official Moroccan educational guidelines. The empirical part is carried out using a questionnaire addressed to teachers and a test dedicated to pupils in order to study the experimental practices of teachers and its effects on students' learning of certain physical concepts. According to teachers, almost of the experimental activities (90\%) designed in the school textbook are not carried out, due to the lack of experimental material and overcrowding of the classes; which affects the completion of the programmed experiments and teachers' experimental practices. The experimental activities rarely performed take the form of lesson experiences, carried out by the teacher himself. Information and Communication Technologies (ICT) are not widely used by teachers, who believe that these technologies would be a good way to complement experiences. The analysis of the responses of pupils showed that they do not master fundamental concepts in the physical sciences when they do not have opportunity to observe in practice or in their life objects related to these concepts.
\end{abstract}

Keywords: experimental activity, textbook, teaching, physical sciences

\title{
1. Introduction
}

L'enseignement des sciences, en particulier les sciences physiques, est un enjeu stratégique vu son rôle de plus en plus important dans la société (Taoufik, 2016). Le développement de l'esprit scientifique chez les apprenants est ainsi un objectif majeur de l'enseignement des sciences, qui devait être visé dès les premières années scolaires, et consolidé au fur et à mesure que l'élève progresse dans ses études. La didactique des sciences physiques s'articule autour d'une série d'hypothèses de départ relatives aux processus d'apprentissage, aux caractéristiques de l'enseignement, et à la nature de la science, en vue d'améliorer l'enseignement-apprentissage des sciences physiques qui se développe dans un cadre défini par le genre du curriculum, les contraintes matérielles vécues dans les conditions réelles de travail, et par les conceptions des enseignants quant à leur travail (Martinand, 1994 ; Louden et Wallace, 1994). 
Comme les pays émergents, le Maroc a fourni des efforts pour améliorer la qualité de l'éducation et assurer sa généralisation. Des réformes ont été entamés depuis l'année 1999, notamment la charte nationale de l'éducation et de la formation (COSEF ; 1999), le plan d'urgence (2009-2013), la vision stratégique de la réforme 2015-2030 (CSEFRS ; 2014). Ces réformes visaient la généralisation de l'enseignement, la restructuration des cycles de l'éducation (secondaire collégial en trois ans et secondaire qualifiant en 3 ans), l'amélioration de sa qualité, la refonte pédagogique et la rénovation des programmes. Le programme GENIE (2009-2013) avait aussi pour objectif la généralisation et l'intégration des technologies d'information et de communication (TIC) dans le système de l'éducation et de la formation.

La rénovation des programmes et des manuels scolaires pourrait élucider les conditions de travail au collège concernant l'enseignement des sciences physiques, du point de vue chercheurs qui apprécient la signification de matériels et de décisions des enseignants, la codification et l'analyse des pratiques pédagogiques et des activités didactiques (expérimentales et documentaires). Cela devrait aider les enseignants à prendre conscience de la cohérence de leurs choix, de mieux mettre en valeur les matériels et les manuels scolaires, et engendrer la rationalisation des réorganisations possibles de leur travail.

L'objet de ce travail est d'une part analyser, par une grille, la place des activités expérimentales dans le manuel scolaire des sciences physiques pour la troisième année du secondaire collégial (MEN, 2015), et d'autre part étudier les pratiques expérimentales des enseignants dans ses classes vis-à-vis des activités conçues dans le programme scolaire. L'influence de ces activités sur l'apprentissage des élèves a été aussi étudiée. Cette analyse est effectuée à travers l'exploitation des réponses d'un questionnaire adressé à un échantillon d'enseignants et un test aux élèves de la troisième année du secondaire collégial.

\section{Problématique}

L'utilisation des activités expérimentales comme contenus et moyens didactique dans l'enseignement des sciences physiques joue un rôle important chez les apprenants ; ces activités aident à la compréhension des phénomènes et concepts physiques à partir d'observations, de manipulations et d'analyse.

La question principale de ce travail est d'examiner si les activités expérimentales conçues dans le manuel scolaire de la troisième année collégiale permettent d'atteindre les objectifs visés, i.e. qu'elles facilitent davantage aux élèves l'apprentissage en vue de développer les compétences requises par le programme. Cette question est déclinée en trois sous questions :

1) Quelle place occupe les activités expérimentales dans le manuel scolaire des sciences physiques de 3 éme année collégiale?

2) Les activités expérimentales prévues par le manuel scolaire sont-elles appliquées dans la pratique? 
3) Les activités expérimentales favorisent-elles davantage l'apprentissage des élèves ?

A noter que la structuration du système éducatif marocain, telle qu'elle a été retenue par la Charte Nationale d'Education et de Formation (COSEF, 1999), est composée de deux degrés : le premier correspond à l'enseignement primaire composé de l'école préscolaire et de l'école primaire ; le second degré s'identifie à l'enseignement secondaire collégial et qualifiant. L'enseignement secondaire collégial, organisé en cycle de trois ans, est ouvert à tous les enfants issus du primaire et détenteurs d'un certificat d'études primaires.

\section{Cadre de références}

Cette partie passe en revue certain concepts liés à la problématique et l'objectif de la présente étude, telles les activités en particulier expérimentales, les manuels scolaires, et les TIC.

\subsection{Activités expérimentales dans les sciences physiques}

Les difficultés des élèves marocains dans l'acquisition des concepts chimiques et physiques ont été étudiés en cycle de secondaire collégial (Benzidia et al., 2021 ; Ouasri et Ravanis, 2020 ; Ouasri, 2018 ; Ouasri, 2017a ; 2017b). Les difficultés qu'éprouvaient les élèves à apprendre des concepts abstraits lors de l'enseignement apprentissage, y compris la résolution de problèmes, ont été expliquées par les conceptions alternatives erronées, et les applications heuristiques chez ces élèves à propos de ces concepts. De l'autre côté, les difficultés des élèves des sciences physiques ont été étudiés en lien avec les activités expérimentales en classes (Giuseppin, 1996 ; Kouhila et Maarouf, 2001 ; Houssaini et al., 2014 ; Taoufik et al., 2016).

L'importance d'une activité est perçue selon le jugement que l'élève porte sur l'utilité de cette activité en lien avec les objectifs qu'il poursuit. En s'appuyant sur les travaux de Dweck (1986, 1989), Viau (1999) distingue les buts d'apprentissage dont l'élève s'engage dans une activité pour acquérir des connaissances et les buts de performance dont l'élève recherche, par le biais de l'activité, une certaine reconnaissance de la part d'autrui. Les deux buts ont été étudiés en relation avec la motivation scolaire (Ames, 1992 ; Viau, 1999 ; Ouasri, 2020). La nature des buts que se fixe un élève constitue une étape essentielle pour mieux comprendre quelle valeur ce dernier va attribuer à une activité spécifique (Viau, 1999). Il est important de considérer le rôle des divers acteurs qui composent les environnements scolaire, familial et social (Tardif, 1992). Ces acteurs peuvent jouer positivement ou négativement dans la valorisation des activités aux yeux de l'élève.

La physique est une science de nature expérimentale qui étudie les phénomènes naturels et leurs évolutions, établit des théories qui permettent de les modéliser, de les expliquer et de les prévoir. Les activités expérimentales dans les sciences physiques sont des activités au cours desquelles les élèves travaillent en groupes sur des questions 
d'enquête guidées et soigneusement conçues. L'utilisation du matériel du laboratoire fournit aux élèves les moyens de collecter des données et de faire des simulations de données recueillies ; ce qui permet de poser des questions et prendre des décisions qui mènent à un apprentissage considéré comme étant une découverte. Au cours de l'expérience, les élèves et (ou) l'enseignant collectent des données et émettent des observations et des hypothèses. L'enseignant anime, facilite la compréhension, et pose des questions permettant d'attirer l'attention des apprenants sur des résultats des activités expérimentales. Celles-ci diffèrent des démonstrations en classe parce que les élèves y participent à la collecte de données ou via les observations. Néanmoins, tout comme dans une démonstration en classe interactive, les élèves impliqués dans des activités expérimentales peuvent être invités à faire des prédictions et à réfléchir à leurs observations.

\subsection{Stratégies d'apprentissage par les activités expérimentales}

$\mathrm{Au}$ collège, on note que le volume horaire alloué aux thèmes du programme n'est pas spécifiquement dédié aux travaux pratiques, alors que dans l'esprit des concepteurs des programmes, l'expérimentation des élèves tient une large place dans les différents thèmes rencontrés. Cependant l'impossibilité de pratiquer des groupes à effectifs allégés rend difficiles les manipulations des élèves de collèges. Ainsi, on pourrait s'étonner que ces élèves pensent que c'est en sciences physiques que les expériences sont plus utiles.

Lorsque les apprentissages ne s'appuient pas sur la pratique expérimentale, l'enseignement des sciences physiques n'apporte rien à la formation pratique ni à la capacité de comprendre le monde, voire d'agir sur lui. En l'absence de support expérimental, les concepts sont présentés sous forme abstraite, et les exercices d'évaluation ne portent que sur l'aptitude à formaliser et les confrontations avec les situations réelles n'y sont pas assez nombreuses. Les rôles des expériences dans la physique savante et à travers les manuels dans la physique scolaire avaient été analysées (Kouhila et Maarouf, 2001 ; Taoufik, 2016). Dans la physique qui s'enseigne, le rôle de l'expérience est souvent réduit à l'illustration des concepts qui sont imposés aux élèves. La présente étude porte sur le rôle des expériences de cours proposées dans le manuel scolaire de la troisième année secondaire collégial marocain. Il s'agit des expériences dont les rôles sont définis dans les programmes scolaires, et réalisées par l'enseignant, qui parfois désigne un élève pour manipuler sous son contrôle. Ces expériences permettent aux enseignants de montrer que les phénomènes de laboratoire ne sont pas différents de ceux observés dans la nature ou mis en œuvre dans les dispositifs technologiques.

Adaptées au contenu du cours, les expériences de cours visibles directement ou par projection constituent une première approche qualitative du concept que l'on se propose d'aborder. Pour les élèves, cette approche est indispensable pour donner du sens à ce concept. Ces expériences devraient étonner, amorcer un questionnement, aider à formuler des hypothèses et poser une problématique; ce qui permet à l'enseignant d'attirer l'attention des élèves sur un aspect d'un phénomène qui pourrait leur échapper, 
et donc de leur apprendre à observer. Aussi, ces expériences donnent aux élèves l'occasion d'apprendre à argumenter pour défendre leur point de vue.

Dans les expériences de cours, la méthode inductive désignée sous le nom de « méthode de redécouverte » et largement utilisée, est fortement critiquée (Gohau, 1989 ; Robardet et Guillaud, 1993), car elle impose la théorie et prétend la déduire d'une expérience que les élèves ont vue. L'élève est donc trompé si on lui laisse croire qu'il a prouvé ou qu'il a découvert la théorie.

L'enseignement expérimental des sciences-physiques, déjà influencé par les nouvelles technologies, est désormais assisté par ordinateur (EXAO). La nouvelle tendance de l'enseignement de sciences-physiques est de placer l'apprenant dans une situation-problème, construite autour d'un problème concret présentant un caractère énigmatique ; ce qui permet aux élèves de formuler des hypothèses et donc dévoiler leurs représentations, concevoir un protocole expérimental, réaliser les expériences pouvant tester leurs hypothèses. La confrontation des résultats obtenus avec celles attendus permet de valider ou d'infirmer les hypothèses. Cette approche de résolution implique certaines étapes : formuler la situation-problème, émettre et vérifier des hypothèses, et interpréter les résultats. La phase d'interprétation est décisive dans la construction du savoir par l'apprenant, car elle lui permet de mettre en relation ses connaissances antérieures et nouvelles. Joshua (1989) indique que l'expérience scientifique ne prendra tout son sens qu'avec une situation problématique de départ. Cette approche vise l'apprentissage de la démarche expérimentale, voire largement la démarche scientifique.

\subsection{Présentation générale du manuel scolaire}

Sans pour autant entrer dans les définitions des manuels scolaires, on peut dire depuis la fin du 19e siècle, le terme manuel désigne les ouvrages qui présentent les connaissances exigées par les programmes scolaires (Claude et al., 2005). Le manuel scolaire permet la mise en texte du contenu du programme scolaire, i.e. qu'il présente, développe et interprète le programme à travers un contenu conçu dans et pour un cadre scolaire déterminé. Dans ce sens, le manuel scolaire représente ainsi un niveau spécifique dans le modèle de transposition didactique.

Dans le contexte marocain, l'élaboration des manuels scolaires s'inscrit dans une perspective visant à élargir et redynamiser l'offre scolaire pour mieux répondre aux besoins des élèves en matière d'apprentissage dans l'enseignement secondaire collégial. Les manuels ont été conçus selon les programmes et les orientations pédagogiques du ministère de l'Education Nationale, de la Formation Professionnelle, de l'Enseignement Supérieur et de la Recherche Scientifique. Le manuel scolaire de la troisième année du secondaire collégial pour les sciences physiques est donc présenté selon une progression cohérente et harmonisée de leçons réparties en trois grands domaines : les matériaux, mécanique et l'électricité (Tableau 1). 
Tableau 1 : Présentation générale du manuel scolaire étudié

\begin{tabular}{|c|c|}
\hline Titre du manuel & Univers plus dans les sciences physiques \\
\hline Année d'édition & 2019 \\
\hline Nombre de pages & 240 \\
\hline Titres des grands domaines & $\begin{array}{ll}\text { - } & \text { Les matériaux } \\
\text { - } & \text { Mécanique } \\
\text { - } & \text { Electricité } \\
\end{array}$ \\
\hline
\end{tabular}

\section{Méthodologie}

La méthodologie adoptée dans cette étude consiste dans un premier temps à effectuer une analyse des principales activités pour chaque leçon du manuel scolaire des sciences physiques de la troisième année du secondaire collégial pour identifier l'importance des activités expérimentales dans le manuel scolaire par rapport aux activités documentaires (Annexe 1). Ensuite, un questionnaire est adressé aux enseignants des sciences physiques de la troisième année du secondaire collégial (Annexe 2) pour collecter des données sur la mise en œuvre par les enseignants des activités expérimentales prévues par le manuel scolaire. Enfin, un test est adressé aux élèves pour étudier la compréhension par les élèves de certains concepts en lien avec l'observation des objets rattachés à ces concepts (Annexe $3)$.

\subsection{Grille d'analyse du manuel scolaire}

Dans le manuel scolaire, des activités pratiques ou documentaires sont prévus, pour chaque leçon, comme des supports des apprentissages. Ces activités sont conçues en vue de structurer les l'apprentissage des notions fondamentales en termes d'objectifs et compétences visés. La grille d'analyse préparée consiste à identifier et classer les activités expérimentales et documentaires dans chaque leçon avec les objectifs attendus. Des remarques sont notées sur la place donnée à ces activités. Ensuite le nombre et le pourcentage des activités expérimentales par rapport aux activités documentaires seront calculés pour tirer les conclusions (Tableau 2, Annexe 1).

\subsection{Questionnaire pour enseignants}

L'échantillon est constitué de 20 enseignants des sciences physiques, répartis sur cinq collèges appartenant à la Direction provinciale de l'éducation nationale de Kénitra (Tableau 3). Ces enseignants ont plus de 5 ans d'expérience. Le questionnaire destiné aux enseignants (annexe 2) porte sur quatre axes : i) l'illustration et la structuration des activités expérimentales dans le manuel scolaire, ii) le volume horaire alloué aux sciences physiques, la disponibilité et l'équipement des laboratoires aux collèges, iii) la pratique expérimentale et l'implication des élèves dans les activités expérimentales, et iv) l'utilisation des TIC dans l'enseignement-apprentissage aux collèges (questions 10-12). Le questionnaire a été adressé aux enseignants concernés, au cours de l'année scolaire 2020/2021. 
Tableau 3 : Répartition de l'échantillon des enseignants par collège

\begin{tabular}{|l|c|}
\hline Nom du collège & Nombre d'enseignants \\
\hline Al Hariri & 4 \\
\hline Al Khawarizmi & 4 \\
\hline Ibn Khaldoun & 4 \\
\hline Al Gharb & 4 \\
\hline Allal Ben Abdellah & 4 \\
\hline Total & $\mathbf{2 0}$ \\
\hline
\end{tabular}

\subsection{Test adressé aux élèves}

Un test a été administré et adressé à 142 élèves de la 3ème année collégiale répartis sur quatre collèges, appartenant à la Direction provinciale de de Kénitra. Comme le montre le tableau 4 , les filles représentent $54 \%$ du total contre $46 \% \%$ pour les garçons. Le test vise à déterminer si les élèves maitrisent ou non certains concepts fondamentaux de sciences physiques en lien avec l'observation des phénomènes illustratifs de ces concepts. Cela permettra de vérifier l'influence des activités expérimentales sur l'apprentissage des élèves. Le test a été passé dans les différents établissements concernés, au cours de l'année scolaire 2020/2021.

Tableau 4 : Répartition de l'échantillon des élèves selon le genre

\begin{tabular}{|l|c|c|c|}
\hline Nom du collège & Nombre des garçons & Nombre des filles & Total \\
\hline Al Hariri & 15 & 21 & 36 \\
\hline Al Khawarizmi & 17 & 19 & 36 \\
\hline Ibn Khaldoun & 16 & 19 & 35 \\
\hline Al Gharb & 17 & 18 & 35 \\
\hline Total & $\mathbf{6 5}$ & $\mathbf{7 7}$ & $\mathbf{1 4 2}$ \\
\hline
\end{tabular}

\section{Résultats et discussions}

\subsection{Manuel scolaire}

La grille d'analyse fait ressortir une place importante des activités expérimentales comme support d'enseignement par les enseignants et d'apprentissage par les élèves. Comme le montre la Figure 1, les 69 activités du manuel se répartissent entre 45 activités expérimentales, soit $65 \%$, et 24 activités documentaires (35\%). Cette figure illustre une prépondérance des activités expérimentales comme support incontournable de l'enseignement des sciences physiques en $3^{\text {ème }}$ année collégiale. 


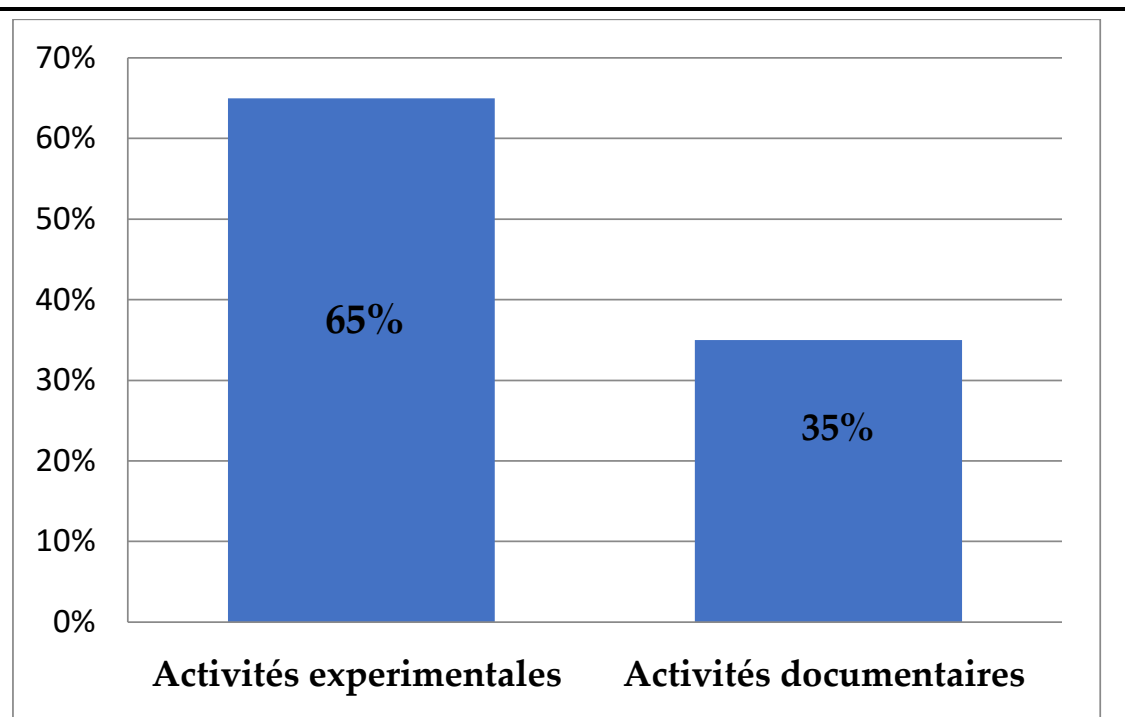

Figure 1 : Relevé des activités expérimentales et documentaires en nombre et en pourcentage du manuel scolaire étudié

Le Tableau 5 présente le nombre des activités expérimentales et documentaires pour les trois domaines de leçons du manuel. Ce qui montre la dominance des activités expérimentales sur les activités documentaires pour le domaine des matériaux et celui de l'électricité. Par contre, dans le domaine de la mécanique, les activités documentaires (au nombre de 9) dépassent les activités expérimentales (au nombre de 7). Les manuels scolaires sont à la fois des ressources pour les enseignants dans les activités de préparation à la conceptualisation et des supports d'appui et d'activités d'exercisation pour les élèves. L'analyse effectuée montre globalement que les activités expérimentales occupent une place importante dans les supports d'enseignement des sciences physiques de la troisième année du secondaire collégiale.

Tableau 1: Nombres des activités expérimentales et documentaires dans chaque partie du manuel scolaire des sciences physiques, troisième année secondaire collégial étudié

\begin{tabular}{|l|c|c|c|}
\hline \multirow{2}{*}{ Domaine } & \multirow{2}{*}{$\begin{array}{c}\text { Nombre de } \\
\text { leçons }\end{array}$} & \multicolumn{2}{c|}{ Nombre d'activités } \\
\cline { 2 - 4 } & 7 & 30 & Documentaires \\
\hline 1. Les matériaux & 4 & 7 & 14 \\
\hline 2. Mécanique & 3 & 8 & 1 \\
\hline 3. Electricité & 14 & 45 & 24 \\
\hline Total & & $\mathbf{6 5} \%$ & $\mathbf{3 5 \%}$ \\
\hline Total des activités $\mathbf{( \% )}$ & & & 9 \\
\hline
\end{tabular}

La grille d'analyse (Annexe 1) des activités expérimentales et documentaires dans le manuel scolaire montre que les activités expérimentales sont présentes dans toutes les leçons à l'exception de la leçon 7 (Dangers de quelques matériaux) et la leçon 9 (Actions mécaniques-Forces) dont on trouve l'analyse documentaire au lieu des activités expérimentales (Tableau 1, Annexe 1). Dans la leçon 7, l'absence des activités expérimentales pourrait être compréhensible, car elle pourrait être due aux mesures préventives, de sécurité et de prudence, vu le caractère dangereux des produits étudiés 
(déchets des matériaux non dégradables). L'objectif de cette leçon est limité à reconnaître les dangers de déchets des matériaux non dégradables, connaître quelques méthodes de gestion et de recyclage des déchets, et prendre conscience de l'importance de préserver la santé et l'environnement. Par contre, l'absence totale des activités expérimentales dans la leçon 9 ne nous parait pas justifiée, car les objectifs affichés peuvent être objets d'activités expérimentales; par exemples :

- Connaitre les actions mécaniques et leurs effets ;

- Distinguer une action de contact d'une action à distance ;

- Connaitre et déterminer les caractéristiques d'une force ;

- Savoir mesurer une intensité à l'aide d'un dynamomètre ;

- Savoir représenter une force en choisissant une échelle convenable.

Néanmoins, le manuel scolaire donne globalement une place importance aux activités expérimentales comme support de l'enseignement des sciences physiques en troisième année du secondaire collégial. Les activités expérimentales dépassent en nombre les activités documentaires dans le manuel scolaire; le nombre des activités expérimentales est $2 / 3$ des activités documentaires. Ce qui montre la place incontournable réservée par les programmes et les orientations pédagogiques officielles à ce type d'activités, qui facilitent davantage l'apprentissage des principaux concepts dispensés à ce niveau. Les activités expérimentales sont donc considérées comme le meilleur moyen didactique pour comprendre et apprendre les concepts des sciences physiques et atteindre les objectifs des leçons conçus dans le manuel scolaire.

Comme le remarque Millar (2004), dans la plupart des pays, les curriculums introduisent les activités scientifiques avec deux principaux objectifs: 1) éducation scientifique ; 2) apprentissage des principaux concepts. Plusieurs auteurs (Viau, 1999 ; Kouhila et Maarouf, 2001 ; Taoufik, 2016) ont révélé que l'utilisation des activités expérimentales comme moyen didactique dans l'enseignement des sciences physiques joue un rôle important chez les apprenants dans l'acquisition correcte des connaissances et notions relatives aux concepts dispensés dans le programme scolaire collégial.

Néanmoins, l'importance de ces activités dans le manuel n'est pas suffisante. Ces activités sont-elles ou non réalisées dans la pratique ? Ce que nous essayerons de montrer via le questionnaire dédié aux enseignants.

\subsection{Questionnaire adressé aux enseignants}

Le tableau 6 présente les réponses recueillies pour les questions (1-7) du questionnaire adressé aux enseignants.

Pour les activités expérimentales dans le manuel scolaire (question 1), on constate que $40 \%$ des enseignants pensent que les activités expérimentales des cours dans le manuel sont bien illustrées, et que la majorité des enseignants $(60 \%)$ ne voient pas ces activités bien illustrées.

S'agissant de la structuration des activités expérimentales dans le manuel scolaire (question 2), on voit que seulement $25 \%$ des enseignants considèrent que la structuration des cours dans le manuel aide les élèves à construire leurs savoirs et savoir-faire et 
d'accéder à l'autonomie. Les résultats obtenus montrent que la plupart des enseignants ne valorisent pas la qualité pédagogique du manuel scolaire au niveau de la structuration des savoirs et des savoir-faire enseignés. Pour ce qui est du volume horaire, la question 3 montre que $70 \%$ des enseignants pensent que le volume horaire alloué aux sciences physiques est insuffisant.

Tableau 6 : Réponses recueillies pour les questions (1-7) du questionnaire enseignant

\begin{tabular}{|l|c|c|}
\hline Questions & $\begin{array}{c}\text { Oui } \\
\mathbf{( \% )}\end{array}$ & $\begin{array}{c}\text { Non } \\
\mathbf{( \% )}\end{array}$ \\
\hline 1. Trouvez-vous les activités expérimentales bien illustrées dans le manuel scolaire & 40 & 60 \\
\hline $\begin{array}{l}\text { 2. La structuration des activités expérimentales dans le manuel scolaire aide les } \\
\text { élèves à construire leurs savoirs, savoir-faire et d'accéder à l'autonomie }\end{array}$ & 25 & 75 \\
\hline 3. Le volume horaire réservé aux sciences physiques est suffisant ? & 30 & 70 \\
\hline 4. Votre établissement possède un laboratoire des sciences physiques ? & 100 & 0 \\
\hline $\begin{array}{l}\text { 5. Le laboratoire est-il équipé du matériel et des produits nécessaires pour réaliser } \\
\text { toutes les activités expérimentales du manuel ? }\end{array}$ & 10 & 90 \\
\hline $\begin{array}{l}\text { 6. Réalisez-vous toutes les activités expérimentales programmées dans le manuel } \\
\text { scolaire? }\end{array}$ & 10 & 90 \\
\hline 7. Demandez-vous à vos élèves de montrer une expérience? & 90 & 10 \\
\hline
\end{tabular}

Pour la disponibilité des laboratoires dans les collèges (question 4), on constate que tous les enseignants $(100 \%)$ ont déclaré que leurs collèges possèdent un laboratoire. Si tous les enseignants affirment que les collèges possèdent des laboratoires, la question 5 montre que $90 \%$ des enseignants voient que ces laboratoires ne sont pas bien équipés du matériel didactique et des produits nécessaires pour effectuer toutes les activités expérimentales conçues dans le programme scolaire des sciences physiques. Ainsi, avoir des laboratoires sans tous équipements nécessaires ne permet pas de réaliser les activités expérimentales, et donc ne permet pas d'atteindre les objectifs escomptés des expérimentations. En l'absence du matériel et des produits nécessaires, les enseignants ne peuvent pas réaliser toutes les activités programmées dans le manuel. Ce qui est confirmé par la question 6, selon laquelle seul $10 \%$ des enseignants affirment réaliser les activités expérimentales programmées dans le manuel scolaire lors de l'enseignement-apprentissage des concepts fondamentaux en sciences physiques au niveau de la troisième année du secondaire collégiale. De la question 7 , on constate que $90 \%$ des enseignants demandent aux élèves de monter une expérience, le cas échéant.

Cependant, l'absence du matériel et des produits nécessaires n'est pas la seule cause qui fait que les enseignants ne réalisent pas les activités programmées dans le manuel. À ce propos, les figures (2-3) présentent les réponses recueilles en termes de pourcentage pour les questions (8-9) du questionnaire adressé aux enseignants. La figure 2 montre que les enseignants évoquent d'autres raisons qui font que toutes les activités expérimentales programmées dans le manuel scolaire ne soient pas réalisées. Si environ $40 \%$ des enseignants attribuent la non réalisation des activités expérimentales au manque de matériels et produits nécessaires, alors $40 \%$ des enseignants déclarent ne pas réaliser ces activités à cause du surnombre des élèves en classe. En revanche, 20\% des enseignants 
signalent qu'ils ne réalisent pas toutes les activités expérimentales du manuel à cause du manque de temps.

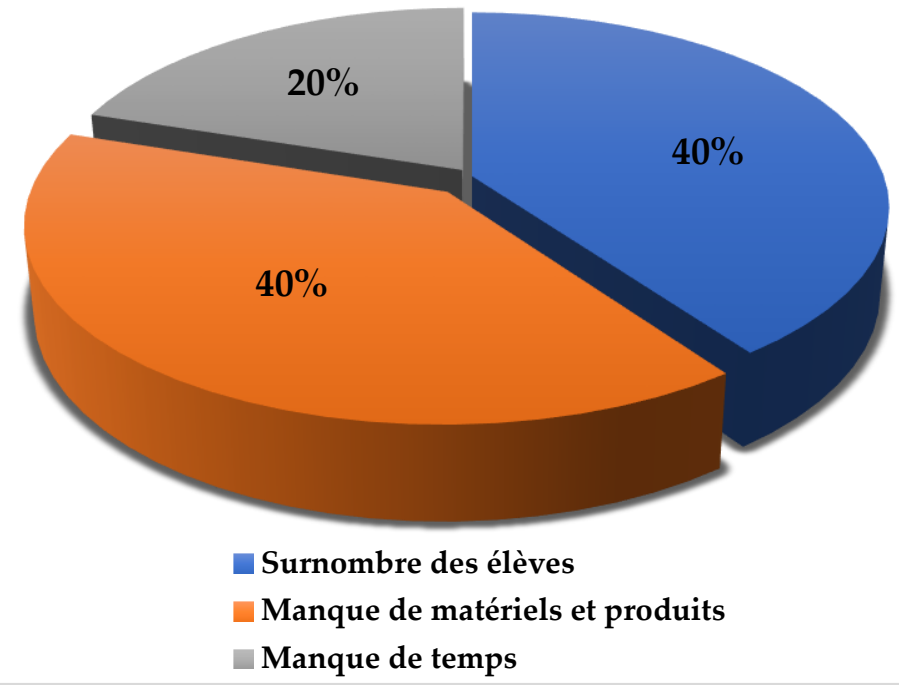

Figure 2 : Raisons évoquées par les enseignants concernant la non réalisation des activités expérimentales

Les réponses collectées sur la réalisation des expériences en classe auprès des enseignants (Figure 3) montrent que les activités expérimentales se font essentiellement sous forme des expériences de cours avec un pourcentage de $65 \%$, les expériences sous forme de TP-cours ont été réalisées avec $25 \%$, et celles sous forme de TP autonome ont été réalisées à $10 \%$ par les élèves organisés en petits groupes.

Il semble que les enseignants choisissent de faire des expériences de cours au lieu des TP à causes de plusieurs raisons que l'on peut dégager de l'analyse des réponses recueillies à savoir :

- le manque du matériel dédié aux activités expérimentales qui impose le choix des expériences de cours,

- le sureffectif des classes, qui affecte l'organisation des activités expérimentales, et le nombre de matériel et les quantités des produits chimiques disponibles (à $10 \%$ ) ne soient pas suffisantes,

- et l'insuffisance de l'horaire imparti aux sciences physiques et à la lourdeur des programmes 


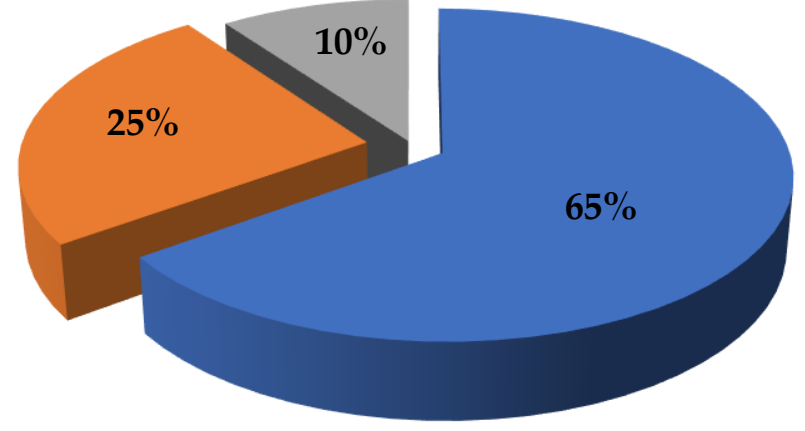

Expériences de cours $\square$ TP-cours $\square$ TP autonome

Figure 3 : Formes des activités expérimentales réalisées par les enseignants

Le Tableau 7 récapitule les réponses recueillies pour les questions (10-12) portant sur l'utilisation des technologies d'information et de communication (TIC) dans l'enseignement apprentissage des sciences physiques en troisième année du secondaire collégial.

Tableau 7 : Réponses recueillies pour les questions (1-7) du questionnaire enseignant

\begin{tabular}{|l|c|c|}
\hline Questions & $\begin{array}{c}\text { Oui } \\
\mathbf{( \% )}\end{array}$ & $\begin{array}{c}\text { Non } \\
\mathbf{( \% )}\end{array}$ \\
\hline 10. Utilisez-vous les TIC & 30 & 70 \\
\hline $\begin{array}{l}\text { 11. A votre avis, la simulation peut réduire les difficultés de l'acquisition des } \\
\text { concepts étudiés en sciences physiques }\end{array}$ & 70 & 30 \\
\hline 12. A votre avis, les TIC peuvent substituer les expériences réelles & 20 & 80 \\
\hline
\end{tabular}

Il s'est avéré de la question (10) que l'utilisation de ces technologies reste faible, puisque seulement $30 \%$ des enseignants déclarent avoir utilisé les TIC dans l'enseignement apprentissage des sciences physiques à ce niveau collégial. La faible utilisation des TIC est en contradiction avec l'intérêt que les enseignants donnent à la simulation ; la question 11 montre que les enseignants pensent que la simulation peut réduire les difficultés de l'acquisition des concepts physiques et chimiques étudiés par les élèves. Quant à la question (12) sur la substitution des expériences réelles par les TIC, l'analyse des réponses collectées montre que $80 \%$ des enseignants ne voient pas que les TIC constituent une alternative pour substituer aux expériences réelles.

La faible utilisation des TIC semble être en contradiction avec l'intérêt porté par les enseignants aux TIC, car 70\% des enseignants pensent que la simulation peut réduire les difficultés de l'acquisition des concepts étudiés. L'intégration de la simulation pourrait motiver les élèves de construire leurs propres savoirs grâce à la visualisation des phénomènes (Ouasri, 2019). Sur un autre registre, la majorité des enseignants (80\%) n'approuvent pas la substitution des expériences réelles par les TIC. Ces résultats ne sont pas en cohérence avec les objectifs fixés par les politiques publique «stratégie Maroc 
numérique » qui accorde à l'École un rôle prometteur dans la diffusion des technologies de l'information et de la communication, et l'intégration de ces technologies sur le renouveau et le progrès de l'École marocaine, ainsi que les recommandations évoquées par le Conseil Supérieur de l'Education, de la Formation et de la Recherche Scientifique (CSEFRS, 2014).

\subsection{Test adressé aux élevés}

La maitrise de certains concepts fondamentaux de la physique-chimie par les élèves de la troisième année du secondaire collégiale, a été étudiée par le test présenté dans $l^{\prime}$ annexe 2. Les figures $3(a-b)$, ainsi que le tableau 2 présentent les résultats obtenus via ce test.
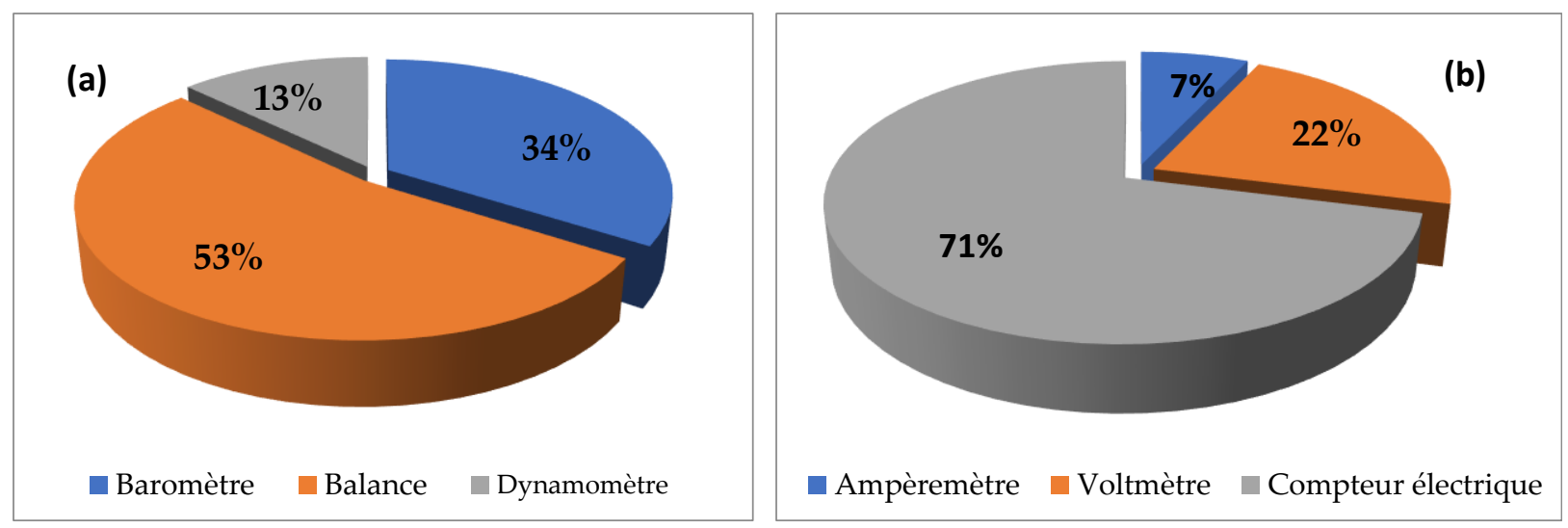

Figure 3 : Réponses des élèves en (\%) concernant l'appareil qui mesure :

a) le poids, b) l'énergie électrique

La Figure 3.a. montre que la majorité des élèves ne savent pas quel est le vrai appareil qui mesure le poids. En effet, seulement $34 \%$ ont répondu que c'est le dynamomètre, tandis que $53 \%$ ont répondu que c'est la balance, et $13 \%$ ont choisi le baromètre. La Figure 3.b. montre que la majorité des élèves (71\%) connaissent l'appareil qui mesure l'énergie électrique.

Le Tableau 2 présente les réponses des élèves (oui ou non) sur les questions 3-6 relevant du test donné dans l'annexe 2. Pour la nature du poids (question 3), il s'est avéré que $63.3 \%$ des élèves ont réussi à répondre que le poids est une force, alors que $36.6 \%$ des élèves n'arrivent pas à comprendre que le poids est une force. Concernant la question 4 portant sur la solution acide $-\mathrm{pH}$, les élèves répondant correctement par « la solution acide a un pH inférieur à 7 » constituent $45 \%$ de l'échantillon testé, alors que $55 \%$ des élèves considèrent que la solution acide a un $\mathrm{pH}$ supérieur à 7. Par ailleurs, les élèves testés connaissent en majorité ( $81 \%$ ) que la corrosion du fer dans l'air est une réaction d'oxydation (question 5). De la question 6, on constate que $70.6 \%$ des élèves considèrent que la combustion n'est pas une réaction chimique, ce qui montre que la majorité des élèves ne maitrisent pas bien le concept de réaction chimique.

Les résultats obtenus montrent que les apprenants sont confus au sujet de certains indicateurs qui mesurent la quantité d'une grandeur, que ce soit pour la similitude du 
concept avec l'appareil utilisé ou la similitude de certains concepts. Ce problème peut être dû au manque des activités expérimentales, comme l'ont montré les réponses recueillies des enseignants. Cependant, le nombre moyen d'apprenants qui a pu connaître certains concepts lorsqu'ils ont l'occasion de les observer met en évidence le rôle des activités expérimentales dans la construction des concepts physiques et chimiques chez les apprenants.

Tableau 2 : Réponses des élèves sur les questions 3-6 du test concernant le poids, la solution acide, la corrosion du fer, et la combustion

\begin{tabular}{|l|c|c|}
\hline Questions & Oui (\%) & Non (\%) \\
\hline 3. Le poids est-il une force ? & 63.4 & 36.6 \\
\hline 4. La solution acide a un $\mathrm{pH}>7$ ? & 55 & 45 \\
\hline 5. La corrosion du fer dans l'air est-elle une oxydation? & 81 & 19 \\
\hline 6. La combustion est-elle une réaction chimique? & 29.4 & 70.6 \\
\hline
\end{tabular}

\section{Conclusion}

$\mathrm{Au}$ terme de ce travail exploratoire, nous pouvons conclure que les activités expérimentales occupent une place importante dans le manuel scolaire d'enseignement des sciences physiques de la troisième année du secondaire collégial au Maroc. L'utilisation des expériences réelles, comme moyen didactique, dans l'enseignement des sciences physiques semble être importante dans l'acquisition des concepts physiques et chimiques chez les apprenants.

Selon les réponses des enseignants, les activités expérimentales proposées dans le manuel scolaire sont faiblement réalisées; le cas échant, réalisées sous forme des expériences de cours par l'enseignant lui-même. Les enseignants faisaient face au manque de matériel expérimental, au sureffectif des classes, et à l'insuffisance du temps, qui fait que le taux de réalisation des expériences est faible.

L'utilisation des technologies d'information et de communication (TIC) reste aussi faible; ce qui est en contradiction avec l'intérêt porté par les enseignants à ces technologies. Ils pensaient que la simulation peut faciliter l'acquisition des concepts étudiés, en motivant davantage les élèves à construire leurs propres savoirs, et que les TIC seraient un bon moyen pour complémenter les expériences réelles.

\section{About the Authors}

Mohammed Abid (Corresponding Author), Centre Régional des Métiers de l'Education et de la Formation, Rabat, Maroc. M. Mohammed Abid has received his PhD in Applied Chemistry from Ibn Tofail University, Kenitra, Morocco in 2000. He had published many researches covering chemistry and science didactic fields in indexed international journals. Currently, his research interest focused as well as on chemistry and science didactic. ORCID: orcid.org/0000-0002-7427-2215, E-mail: profabidmed@gmail.com, abid.mohammed@uit.ac.ma 
Amina Boulahoual, Centre Régional des Métiers de l'Education et de la Formation, Rabat, Maroc. Mrs. has received his $\mathrm{PhD}$ in mathematical physics from Ibn Tofail University, Kenitra, Morocco in 2009. She had published many researches covering physics and science didactic fields in indexed international journals. Currently, his research interest focused on didactic of sciences. E-mail: boulahoual@gmail.com

M. Abdeslem Daif has received his specialized Master in physics and chemistry teaching and training from Ibn Tofail University, Faculty of Sciences, Kenitra, Morocco in 2021 Email: abdeslamdaif@gmail.com

Ali Ouasri, Centre Régional des Métiers de l'Education et de la Formation, Rabat, Maroc. M. Ali Ouasri has received his PhD in Applied Chemistry from Ibn Tofail University, Kenitra, Morocco in 2002. He had published many researches covering chemistry and science didactic fields in indexed international journals. Currently, his research interest focused as well as on chemistry and science didactic. ORCID: orcid.org/0000-0002-71457759. E-mail : aouasri@yahoo.fr

\section{Références}

Ames, C. (1992). Classrooms: Goals, structures, and student motivation. Journal of Educational Psychology, 84(3), 261-271.

Benzidia, B., Ait Alioua, H., Ouasri, A., Abid, M. (2021). Identification des difficultés des apprenants du secondaire collégial marocain dans l'apprentissage des concepts de transformation chimique - transformation physique, European Journal of Education Studies 8(11) 246-265.

Commission Spéciale Education Formation (COSEF), Maroc. (1999). Charte nationale D'éducation et de formation.

Conseil Supérieur de l'Education, Maroc. (2009). Programme national d'Evaluation des Acquis PENA 2008 : Rapport synthétique.

Conseil Supérieur de l'Education, de la Formation et de la Recherche Scientifique (CSEFRS), Maroc. (2014). Pour une école de l'équité de la qualité et de la promotion, Vision stratégique de la réforme 2015-2030.

Dweck, C. S. (1986). Motivational processes affecting learning. American Psychologist, 41(10), 1040-1048.

Dweck, C. S. (1989). Motivation. In A. Lesgold et R. Glaser (dir.), Foundations for a psychology of education (p. 87-136). Hillsdale, NJ : Erlb.

Giuseppin, M. (1996). Place et rôle des activités expérimentales en sciences physiques. Didaskalia, 9, 107-118.

Houssaini, W. I., Hassouni, T., Echalfi, F., Ziali, F. (2014). Importance des expériences dans l'enseignement et l'apprentissage du système nerveux au collège : Etude de cas. European Scientific Journal, 10(28), 155-168.

Karsenti, T., Tchameni, N. (2009). Intégration pédagogique des TIC : Stratégies d'action et pistes de réflexion. Ottawa : Centre de recherches pour le développement international (CRDI). 
Kouhila, M. Maarouf, A. (2001). Approche épistémologique et didactique des fonctions de l'expérience dans la physique savante et scolaire, RES. Academica, 19 (1et2), 9-38.

Louden, W., Wallace, J. (1994). Knowing and teaching science: the constructivist paradox. International Journal of Science Education, 16(6), 649-657.

Martinand, J. L. (1994). La Didactique des Sciences et de la Technologie et la formation des enseignants. Aster, 19, 61-75.

Johsua, S. (1989). Le rapport à l'expérimental dans la physique de l'enseignement secondaire. Aster, (8), 29-53.

Manuel scolaire : Ahmed, S., Mustapha, Z. (2019). Univers plus dans les sciences physiques, troisième année du cycle collégial, Ed. Dar nachr al maarifa. Maroc.

Ministère de l'éducation nationale (MEN), Maroc, (2015). Les orientations pédagogiques des Sciences physiques : cycle collège.

Millar R. (2004). The role of practical work in the teaching and learning of science, High school science laboratories: role and vision, Washington DC, USA: National Academy of Sciences, pp. 1-24.

Johsua, S. et Dupin, J. J. (1993). Introduction à la didactique des sciences et des mathématiques. Paris : PUF.

Ouasri, A. (2017a). Analyse des connaissances des de troisième année du collège marocain en activités de résolution de problèmes de l'électricité (loi d'Ohm, puissance et énergie électrique), European Journal of Education Studies, 3(7) 94-120.

Ouasri, A. (2017b). Analyse des difficultés des élèves marocains de 15-16 ans en résolution de problèmes de mécanique (mouvement et repos, intéractions mécaniques et forces, poids et masse), Review of Mathematic, Sciences and ICT Education, 11(2), 69-92.

Ouasri, A. (2018). Cross-analysis of knowledge and skills in the performance of Moroccan pupils (15-16 years) in solving electricity problems, Journal Plus Education, XIX (1) 289-312.

Ouasri A., Bouatlaoui T. (2019). Motivation et intérêt des élèves de collège marocain pour l'apprentissage des sciences physiques, European Journal of Education Studies, 6(7) 236-256. doi: 10.5281/zenodo.3514627.

Ouasri, A., Ravanis, K. (2020). Apprentissage des élèves de troisième année de collège marocain du concept d'ion en lien avec la trame conceptuelle (atome, molecule, electron, charge); European Journal of Alternative Education Studies, 5(1) 71-94.

Taoufik, M., Abouzaid, A., Moufti, A. (2016). Les activités expérimentales dans l'enseignement des sciences physiques : cas des collèges marocains. European Scientific Journal, 22-190.

Mastafi, M. (2013). Intégration et usages des TIC dans le système éducatif marocain : attitudes des enseignants de l'enseignement primaire et secondaire. Portique adjectif.net. 28 Avril 2013.

Robardet, G., Guillaud, J-C. (1993). Eléments d'épistémologie et de didactique des sciences physiques. Grenoble, publication de l'IUFM.

Viau, R. (1999). Pour une catégorisation des facteurs environnementaux qui influencent la motivation des élèves en contexte scolaire. Apprentissage et socialisation, 19(2), 65-79. 


\section{Annexe 1}

Tableau 1 : Grille d'analyse des activités d'enseignement dans le manuel scolaire étudié

\begin{tabular}{|c|c|c|c|c|}
\hline \multirow{2}{*}{ Leçon } & \multirow{2}{*}{ Objectifs } & \multicolumn{2}{|c|}{ Activités } & \multirow{2}{*}{ Remarques } \\
\hline & & Expérimentale & Documentaire & \\
\hline 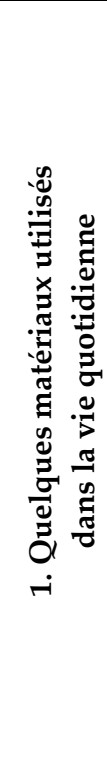 & $\begin{array}{l}\text { - Distinguer les objets } \\
\text { des matériaux qui les } \\
\text { constituent. } \\
\text { - Classer les matériaux } \\
\text { (métaux, verre, plastique) } \\
\text { selon leurs propriétés. } \\
\text { - Connaître les } \\
\text { propriétés de quelques } \\
\text { matériaux. } \\
\text { - Prendre conscience de } \\
\text { l'importance du choix de } \\
\text { matériaux d'emballage. }\end{array}$ & $\begin{array}{l}\text { 1. Conduction } \\
\text { électrique } \\
\text { 2. Conduction de } \\
\text { chaleur } \\
\text { 3. Mesure à l'aide d'une } \\
\text { balance la masse de } \\
\text { différents matériaux } \\
\text { 4. Test de } \\
\text { reconnaissance des } \\
\text { métaux } \\
\text { 5. Mesure à l'aide d'une } \\
\text { balance la masse de } \\
\text { différents métaux } \\
\text { 6. Test de flottabilité } \\
\text { 7. Test de la couleur de } \\
\text { flamme } \\
\text { 8. Test d'identification } \\
\text { du PS et PET }\end{array}$ & $\begin{array}{l}\text { 1. Présentation de } \\
\text { quelques objets et } \\
\text { matériaux utilisés dans } \\
\text { la vie quotidienne } \\
\text { 2. Illustration des } \\
\text { oorigines et de la } \\
\text { famille des objets et } \\
\text { matériaux. } \\
\text { 3. Observation des } \\
\text { photos pour } \\
\text { déterminer les } \\
\text { créitérés du choix d'un } \\
\text { matériau d'emballage }\end{array}$ & $\begin{array}{l}\text { Bonne présence des } \\
\text { activités } \\
\text { expérimentales }\end{array}$ \\
\hline 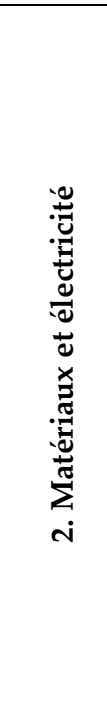 & $\begin{array}{l}\text { - Connaître les } \\
\text { constituants de l'atome. } \\
\text { - Connaître la } \\
\text { signification du numéro } \\
\text { atomique. } \\
\text { - Connaître l'électro- } \\
\text { neutralité de l'atome. } \\
\text { - Classer les ions en ion } \\
\text { monoatomique et ion } \\
\text { polyatomique. } \\
\text { • Écrire la formule d'un } \\
\text { ion connaissant le } \\
\text { nombre d'électrons reçus } \\
\text { ou perdus par l'atome. }\end{array}$ & $\begin{array}{l}\text { 9. Test de vérification } \\
\text { de la conduction d'une } \\
\text { solution }\end{array}$ & $\begin{array}{l}\text { 4. Analyse d'un } \\
\text { document donnant un } \\
\text { aperçu sur l'évolution } \\
\text { des notions se } \\
\text { rapportant à l'atome. } \\
\text { 5. Analyse d'un } \\
\text { document donnant des } \\
\text { atomes pour des } \\
\text { matériaux différents } \\
\text { 6. Analyse d'un } \\
\text { document donnant le } \\
\text { modèle d'atome de } \\
\text { sodium, de chlore et } \\
\text { leur nuage } \\
\text { électronique avant et } \\
\text { après avoir perdu ou } \\
\text { gagné des électrons }\end{array}$ & $\begin{array}{l}\text { Faiblesse des activités } \\
\text { expérimentales }\end{array}$ \\
\hline
\end{tabular}




\begin{tabular}{|c|c|c|c|c|}
\hline 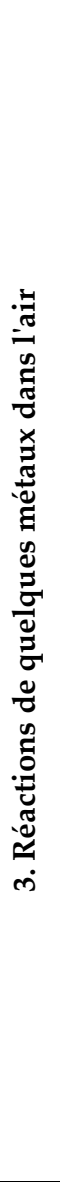 & $\begin{array}{l}\text { - Connaître les facteurs } \\
\text { favorisant l'oxydation du } \\
\text { fer dans l'air humide. } \\
\text { - Connaître quelques } \\
\text { propriétés de la rouille et } \\
\text { savoir protéger le fer } \\
\text { contre la corrosion. } \\
\text { - Expliquer la dé } \\
\text { déférence entre } \\
\text { l'oxydation dans l'air du } \\
\text { fer et de l'aluminium. } \\
\text { - Écrire l'équation de la } \\
\text { réaction correspondant à } \\
\text { la formation des oxydes } \\
\text { Al Al } 2 \mathrm{O}_{3} \text { et Fe Fe2O3. } \\
\text { - Reconnaître les } \\
\text { produits de combustion } \\
\text { de quelques matériaux } \\
\text { organiques dans le } \\
\text { dioxygène de l'air et } \\
\text { déduire le type d'atomes } \\
\text { constituant ces } \\
\text { matériaux. } \\
\text { - Connaître les dangers } \\
\text { de combustion de } \\
\text { matériaux organiques et } \\
\text { leurs effets sur la santé et } \\
\text { l'environnement. }\end{array}$ & $\begin{array}{l}\text { 10. Test d'oxydation du } \\
\text { fer dans l'air humide } \\
\text { 11. Test d'oxydation de } \\
\text { l'aluminium dans l'air } \\
\text { 12. Test de combustion } \\
\text { du papier } \\
\text { 13. Test de combustion } \\
\text { du plastique }\end{array}$ & $\begin{array}{l}\text { 7. Analyse d'un } \\
\text { document sur } \\
\text { l'oxydation du fer et } \\
\text { comment le protéger } \\
\text { contre la corrosion } \\
\text { 8. Analyse d'un } \\
\text { document donnant les } \\
\text { dangers de } \\
\text { combustion des } \\
\text { matériaux organiques }\end{array}$ & $\begin{array}{l}\text { Présence des activités } \\
\text { expérimentales }\end{array}$ \\
\hline 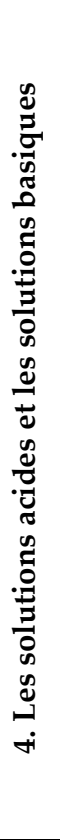 & $\begin{array}{l}\text { - Savoir la signification } \\
\text { du pH. } \\
\text { - Utiliser papier pH et } \\
\text { pH pH-mètre pour } \\
\text { mesurer le pH d'une } \\
\text { solution. } \\
\text { - Classer les solutions } \\
\text { acide, basique et neutre } \\
\text { selon la valeur du pH. } \\
\text { - Connaître à travers des } \\
\text { étiquettes, quelques } \\
\text { dangers des solutions } \\
\text { acides et basiques, et } \\
\text { appliquer les précautions } \\
\text { préventives lors de leur } \\
\text { utilisation. } \\
\text { - Reconnaitre la dilution } \\
\text { d'une solution acide ou } \\
\text { basique et son effet sur la } \\
\text { valeur pH. }\end{array}$ & $\begin{array}{l}\text { 14. Mesure à l'aide du } \\
\text { papier } \mathrm{pH} \\
\text { 15. Mesure à l'aide d'un } \\
\text { pH métré } \\
\text { 16. Test d'identification } \\
\text { des caractéristiques } \\
\text { d'une solution acide et } \\
\text { une solution basique } \\
\text { 17. Test de dilution } \\
\text { d'une solution acide } \\
\text { 18. Test de dilution } \\
\text { d'une solution basique }\end{array}$ & $\begin{array}{l}\text { 9. Analyse d'un } \\
\text { document donnant la } \\
\text { signification du pH } \\
\text { 10. Analyse d'un } \\
\text { document représentent } \\
\text { les étiquettes qu'on } \\
\text { retrouve sur } \\
\text { l'emballage d'un } \\
\text { produit détartrant, } \\
\text { d'un produit } \\
\text { déboucheur ou des } \\
\text { produits à caractère } \\
\text { acide ou basique }\end{array}$ & $\begin{array}{l}\text { Bonne présence des } \\
\text { activités } \\
\text { expérimentales }\end{array}$ \\
\hline
\end{tabular}




\begin{tabular}{|c|c|c|c|}
\hline 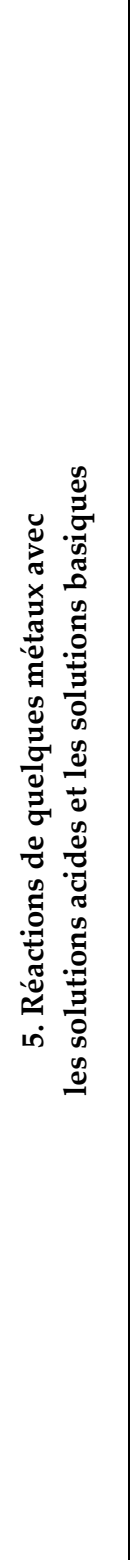 & $\begin{array}{l}\text { - Connaître l'action d'une } \\
\text { solution d'acide } \\
\text { chlorhydrique sur les } \\
\text { métaux fer, cuivre, zinc et } \\
\text { aluminium. } \\
\text { - Écrire l'équation bilan } \\
\text { de la réaction de l'acide } \\
\text { chlorhydrique sur les } \\
\text { métaux fer, cuivre, zinc et } \\
\text { aluminium. } \\
\text { - Connaître l'action d'une } \\
\text { solution d'hydroxyde de } \\
\text { sodium sur les métaux } \\
\text { fer, cuivre, zinc et } \\
\text { aluminium. }\end{array}$ & $\begin{array}{l}\text { 19. Test d'acidité } \\
\text { 20. Test au nitrate } \\
\text { d'argent. } \\
\text { 21. Test d'une solution } \\
\text { d'acide chlorhydrique } \\
\text { sur le fer } \\
\text { 22. Ajoute de solution } \\
\text { nitrate d'argent et } \\
\text { solution d'hydroxyde } \\
\text { de sodium à la solution } \\
\text { finale d'acide } \\
\text { chlorhydrique sur le fer } \\
\text { 23. Test d'une solution } \\
\text { d'acide chlorhydrique } \\
\text { sur les métaux cuivre, } \\
\text { zinc et aluminium } \\
24 \text {. Ajoute de solution } \\
\text { nitrate d'argent et } \\
\text { solution d'hydroxyde } \\
\text { de sodium à la solution } \\
\text { finale d'acide } \\
\text { chlorhydrique sur les } \\
\text { métaux zinc, } \\
\text { l'aluminium et le } \\
\text { cuivre } \\
\text { 25. Test de basicité } \\
\text { pour une solution } \\
\text { d'hydroxyde de } \\
\text { sodium } \\
26 . \text { Test d'une solution } \\
\text { d'hydroxyde de } \\
\text { sodium sur le zinc et } \\
\text { l'aluminium. } \\
27 . \text { Test d'une solution } \\
\text { d'hydroxyde de } \\
\text { sodium sur le fer et le } \\
\text { cuivre }\end{array}$ & $\begin{array}{l}\text { Bonne présence des } \\
\text { activités } \\
\text { expérimentales }\end{array}$ \\
\hline 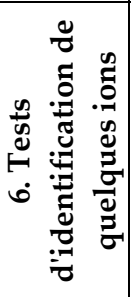 & $\begin{array}{l}\text { - Connaître les tests } \\
\text { d'identification des ions } \\
\left(\mathrm{Fe}^{2+} ; \mathrm{Fe}^{3+} ; \mathrm{Zn}^{2+} ; \mathrm{Al}^{3+} ;\right. \\
\left.\mathrm{Cu}^{2+} ; \mathrm{Cl}\right) \text {. } \\
\text { - Écrire l'équation de } \\
\text { précipitation de ces ions. }\end{array}$ & $\begin{array}{l}\text { 28. Test d'identification } \\
\text { des ions : } \mathrm{Fe}^{2+} ; \mathrm{Fe}^{3+} \text {. } \\
\text { 29. Test d'identification } \\
\text { des ions : } \mathrm{Zn}^{2+} ; \mathrm{Al}^{3+} ; \\
\mathrm{Cu}^{2+} \text {. } \\
\text { 30. Test d'identification } \\
\text { d'ion chlorure. }\end{array}$ & $\begin{array}{l}\text { Présence des activités } \\
\text { expérimentales }\end{array}$ \\
\hline
\end{tabular}




\begin{tabular}{|c|c|c|c|c|}
\hline 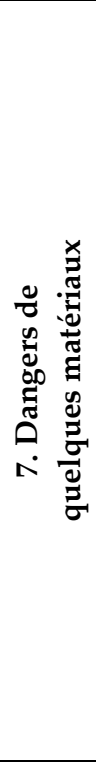 & $\begin{array}{l}\text { - Reconnaître les dangers } \\
\text { de déchets des matériaux } \\
\text { non dégradables. } \\
\text { - Connaître quelques } \\
\text { méthodes de gestion et } \\
\text { de recyclage des déchets. } \\
\text { - Prendre conscience de } \\
\text { l'importance de préserver } \\
\text { la santé et } \\
\text { l'environnement. }\end{array}$ & & $\begin{array}{l}\text { 11. Analyse d'un } \\
\text { document donnant } \\
\text { l'origine des déchets } \\
\text { 12. Analyse d'un } \\
\text { document présentant } \\
\text { quelques aspects au } \\
\text { quotidien lié à la } \\
\text { présence de déchets } \\
\text { 13. Analyse d'un } \\
\text { document sur } \\
\text { comment gérer et } \\
\text { traiter les déchets } \\
\text { 14. Analyse d'un } \\
\text { document donnant } \\
\text { quelques situations en } \\
\text { relation avec } \\
\text { l'utilisation et la } \\
\text { gestion des déchets }\end{array}$ & $\begin{array}{l}\text { Absence des activités } \\
\text { expérimentales }\end{array}$ \\
\hline 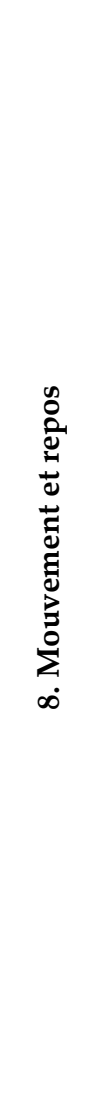 & $\begin{array}{l}\text { - Savoir les notions : } \\
\text { référentiel et trajectoire. } \\
\text { - Connaitre l'état de } \\
\text { mouvement et de repos } \\
\text { d'un solide par rapport à } \\
\text { un référentiel. } \\
\text { - Distinguer les deux } \\
\text { types de mouvements } \\
\text { (Translation et rotation). } \\
\text { - Connaitre l'expression } \\
\text { et l'unité de la vitesse } \\
\text { moyenne et calculer sa } \\
\text { valeur en m/s et km/h. } \\
\text { - Connaitre et } \\
\text { déterminer la nature du } \\
\text { mouvement d'un solide } \\
\text { (uniforme, accéléré, } \\
\text { retardé). } \\
\text { - Connaitre les dangers } \\
\text { de l'excès de vitesse. } \\
\text { - Connaitre quelques } \\
\text { facteurs qui influent sur } \\
\text { la distance d'arrêt. } \\
\text { - Connaitre et appliquer } \\
\text { les règles de sécurité } \\
\text { routière. }\end{array}$ & $\begin{array}{l}\text { 31. Test de mouvement } \\
\text { de translation } \\
\text { 32. Test de mouvement } \\
\text { de rotation }\end{array}$ & $\begin{array}{l}\text { 15. Analyse d'un } \\
\text { document donnant les } \\
\text { notions de référentiel } \\
\text { 16. Analyse d'un } \\
\text { document donnant les } \\
\text { notions de trajectoire } \\
\text { 17. Analyse d'un } \\
\text { document représentent } \\
\text { la vitesse moyenne et } \\
\text { comment la calculer } \\
\text { 18. Analyse d'un } \\
\text { document donnant la } \\
\text { nature de mouvement } \\
\text { d'un solide } \\
\text { 19. Analyse d'un } \\
\text { document représentant } \\
\text { les dangers de la } \\
\text { vitesse et sécurité } \\
\text { routière }\end{array}$ & $\begin{array}{l}\text { Faible des activités } \\
\text { expérimentales. }\end{array}$ \\
\hline
\end{tabular}




\begin{tabular}{|c|c|c|c|c|}
\hline 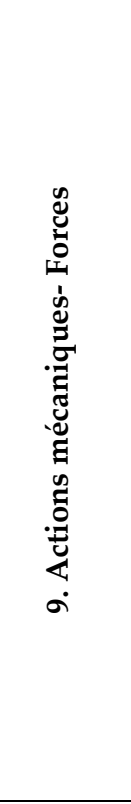 & $\begin{array}{l}\text { - Connaitre les actions } \\
\text { mécaniques et leurs } \\
\text { effets. } \\
\text { - Distinguer une action } \\
\text { de contact d'une action à } \\
\text { distance. } \\
\text { - Savoir qu'une action } \\
\text { mécanique se modélise } \\
\text { par une force. } \\
\text { - Connaitre et } \\
\text { déterminer les } \\
\text { caractéristiques d'une } \\
\text { force. } \\
\text { - Savoir mesurer une } \\
\text { intensité à l'aide d'un } \\
\text { dynamomètre. } \\
\text { - Savoir représenter une } \\
\text { force en choisissant une } \\
\text { échelle convenable. }\end{array}$ & & $\begin{array}{l}\text { 20. Analyse d'un } \\
\text { document sur les } \\
\text { actions mécaniques et } \\
\text { leurs effets } \\
\text { 21. Analyse d'un } \\
\text { document représentent } \\
\text { les différents types } \\
\text { d'action mécanique } \\
\text { 22. Analyse d'un } \\
\text { document sur la } \\
\text { modélisation des } \\
\text { actions mécaniques }\end{array}$ & $\begin{array}{l}\text { Absence des activités } \\
\text { expérimentales }\end{array}$ \\
\hline 完 & $\begin{array}{l}\text { - Connaître et appliquer } \\
\text { la condition d'équilibre. }\end{array}$ & $\begin{array}{l}\text { 33.Test d'équilibre d'un } \\
\text { solide soumis à deux } \\
\text { forces }\end{array}$ & & $\begin{array}{l}\text { Une seule activité } \\
\text { expérimentale }\end{array}$ \\
\hline 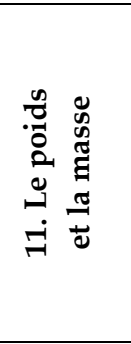 & $\begin{array}{l}\text { - Connaître et } \\
\text { déterminer les } \\
\text { caractéristiques du poids } \\
\text { d'un corps. } \\
\text { - Distinguer poids et } \\
\text { masse d'un corps. } \\
\text { - Connaitre et exploiter } \\
\text { le relation } \mathrm{P}=\mathrm{mg} \text {. } \\
\end{array}$ & $\begin{array}{l}\text { 34. Test du sens et } \\
\text { intensité du poids } \\
\text { 35. Test du point } \\
\text { d'application de la } \\
\text { force poids } \\
\text { 36. Mesure de la masse } \\
\text { 37. Mesure de la force }\end{array}$ & $\begin{array}{l}\text { 23. Analyse d'un } \\
\text { document sur la } \\
\text { différence entre le } \\
\text { poids et la masse }\end{array}$ & $\begin{array}{l}\text { Présence des activités } \\
\text { expérimentales }\end{array}$ \\
\hline 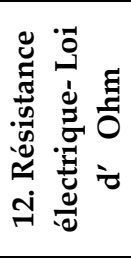 & $\begin{array}{l}\text { - Réaliser, à partir d'un } \\
\text { schéma, un montage } \\
\text { expérimental simple pour } \\
\text { vérifier la loi d'Ohm. } \\
\text { - Connaitre la loi d'Ohm } \\
\text { et l'appliquer. } \\
\end{array}$ & $\begin{array}{l}\text { 38. Mesure la valeur } \\
\text { d'une résistance à l'aide } \\
\text { d'un ohmmètre }\end{array}$ & & $\begin{array}{l}\text { Une seule activité } \\
\text { expérimentale }\end{array}$ \\
\hline 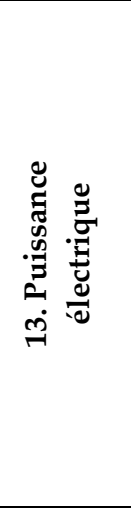 & $\begin{array}{l}\text { - Connaître la puissance } \\
\text { électrique et son unité. } \\
\text { - Connaître les } \\
\text { caractéristiques } \\
\text { nominales d'un appareil } \\
\text { électrique. } \\
\text { - Connaître et appliquer } \\
\text { la relation P = U.I. } \\
\text { - Déterminer la } \\
\text { puissance électrique } \\
\text { consommée par un } \\
\text { appareil de chauffage. }\end{array}$ & $\begin{array}{l}\text { 39. Utilisation d'une } \\
\text { lampe }(12 \mathrm{~V}, 25 \mathrm{~W}) \\
\text { 40. Utilisation d'une } \\
\text { lampe }(12 \mathrm{~V}, 40 \mathrm{~W}) \\
\text { 41. Mesure de la } \\
\text { tension et l'intensité du } \\
\text { courant. } \\
\text { 42. Mesure la puissance } \\
\text { électrique d'un appareil } \\
\text { de chauffage. }\end{array}$ & & $\begin{array}{l}\text { Présence des activités } \\
\text { expérimentales }\end{array}$ \\
\hline
\end{tabular}




\begin{tabular}{|c|c|c|c|c|}
\hline 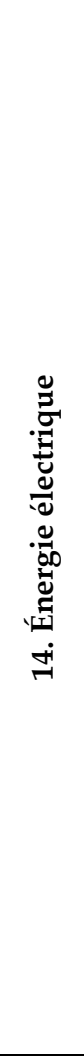 & $\begin{array}{l}\text { - Connaître l'énergie } \\
\text { électrique et ses unités } \\
\text { (Le joule, Le wattheure). } \\
\text { - Connaître et appliquer } \\
\text { la relation E= P.t } \\
\text { - Déterminer l'énergie } \\
\text { électrique consommée } \\
\text { par un appareil de } \\
\text { chauffage. } \\
\text { - Savoir que l'énergie } \\
\text { électrique consommée } \\
\text { par un appareil du } \\
\text { chauffage se transforme } \\
\text { en énergie thermique. } \\
\text { - Savoir le rôle du } \\
\text { compteur électrique dans } \\
\text { une installation } \\
\text { domestique. } \\
\text { •Déterminer l'énergie } \\
\text { électrique consommée } \\
\text { dans une installation } \\
\text { domestique à partir } \\
\text { d'une quittance ou des } \\
\text { donnés d'un compteur } \\
\text { électrique. }\end{array}$ & $\begin{array}{l}\text { 43. Consommation } \\
\text { d'une lampe de } 75 \mathrm{~W} \\
\text { 44. Consommation } \\
\text { d'une lampe de } 150 \mathrm{~W} \\
\text { 45. Mesure de l'énergie } \\
\text { électrique }\end{array}$ & $\begin{array}{l}\text { 24. Analyse d'un } \\
\text { document présentent } \\
\text { des informations dans } \\
\text { une facture } \\
\text { d'électricité }\end{array}$ & $\begin{array}{l}\text { Présence des activités } \\
\text { expérimentales }\end{array}$ \\
\hline 总 & & 45 & 24 & $\begin{array}{l}\text { Le nombre des } \\
\text { activités } \\
\text { expérimentales est 2/3 } \\
\text { des activités } \\
\text { documentaires }\end{array}$ \\
\hline 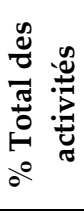 & & $65 \%$ & $35 \%$ & $\begin{array}{l}\text { Les activités } \\
\text { expérimentales sont } \\
\text { présentes la plupart } \\
\text { des activités du } \\
\text { manuel }\end{array}$ \\
\hline
\end{tabular}


Annexe 2 : Questionnaire destiné aux enseignants

Etablissement : ....

Ancienneté dans l'enseignement : ....

1. Trouvez-vous les activités expérimentales bien illustrées dans le manuel

Oui

Non

2. La structuration des activités expérimentales dans le manuel scolaire aide les élèves à construire leurs savoirs, savoir-faire et d'accéder à l'autonomie :

Oui $\square$ Non $\square$

3. Le volume horaire réservé aux sciences physiques est suffisant?

Oui $\square$ Non $\square$

4. Votre établissement possède un laboratoire des sciences physiques?

Oui $\square$ Non $\square$

5. Le laboratoire est-t-il équipé des matériels et des produits nécessaires pour réaliser des toutes les activités expérimentales du manuel?

Oui $\square$ Non $\square$

6. Réalisez-vous tous les activités expérimentales programmées dans le manuel scolaire ?

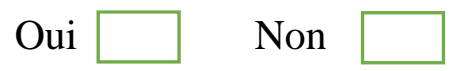

7. Demandez-vous à vos élèves de monter une expérience?

Oui $\square$ Non $\square$

8. Les expériences du manuel sont réalisées sous forme :

Expériences de cours $\square$ TP-cours $\square$ TP autonome $\square$

9. Quelles sont les raisons pour ne pas réaliser toutes les activités expérimentales programmées dans le manuel ?

10. Utilisez-vous les TIC?

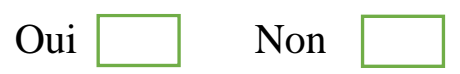

11. A votre avis, la simulation peut réduire les difficultés de l'acquisition des concepts étudiés en sciences physiques:

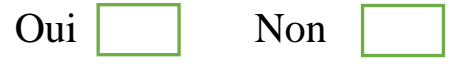

12. A votre avis, les TIC peuvent substituer les expériences réelles?

Oui $\square$ Non $\square$ 
Annexe 3 : Test adressé aux élèves

Etablissement : ....

Sexe : ....

1. Quel appareil mesure le poids ?

Baromètre $\square$ Balance $\square$ Dynamomètre

2. Quel appareil mesure l'énergie électrique?

$$
\text { Ampéremétre } \square \text { Voltmètre } \square \text { Compteur électrique } \square
$$

3. Le poids est-il une force?

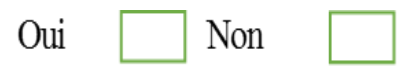

4. La solution acide a un $\mathrm{pH}>7$ ?

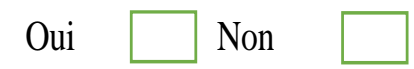

5. La corrosion du fer dans l'air est-elle une oxydation?

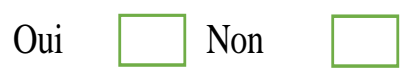

6. La combustion est-elle une réaction chimique?

Oui $\square$ Non 
Creative Commons licensing terms

Authors will retain the copyright of their published articles agreeing that a Creative Commons Attribution 4.0 International License (CC BY 4.0) terms will be applied to their work. Under the terms of this license, no permission is required from the author(s) or publisher for members of the community to copy, distribute, transmit or adapt the article content, providing a proper, prominent and unambiguous attribution to the authors in a manner that makes clear that the materials are being reused under permission of a Creative Commons License. Views, opinions and conclusions expressed in this research article are views, opinions and conclusions of the author(s). Open Access Publishing Group and European Journal of Alternative Education Studies shall not be responsible or answerable for any loss, damage or liability caused in relation to/arising out of conflict of interests, copyright violations and inappropriate or inaccurate use of any kind content related or integrated on the research work. All the published works are meeting the Open Access Publishing requirements and can be freely accessed, shared, modified, distributed and used in educational, commercial and non-commercial purposes under a Creative Commons Attribution 4.0 International License (CC BY 4.0). 